\title{
A response instruction by visual-field interaction: S-R compatibility effect or ?
}

\author{
BILL COTTON and OVID J. L. TZENG \\ University of California, Riverside, California 92521 \\ and \\ CURTIS HARDYCK \\ University of California, Berkeley, California 94720
}

\begin{abstract}
Stimulus-response compatibility effects have been hypothesized to result from a correspondence between the spatial codes associated with the response hand and the stimulus location (i.e., the S-S interpretation of Wallace, 1971) or, alternatively, from a subject's innate tendency to respond in the direction of the source of stimulation (i.e., the S-R interpretation of Craft \& Simon, 1970). Two experiments were conducted to test these two positions. Patterns of response latencies were found that could not be adequately explained by either model.
\end{abstract}

Numerous studies have shown that reaction time (RT) to a signal decreases when the spatial location of the response corresponds to the spatial location of the command signal. The phenomenon has been termed the stimulus (S) and response (R) compatibility effect. This effect has been found both with a visual display (Rabbitt, 1967; Simon \& Wolf, 1963) and with auditory signals (Bertera, Callan, Parsons, \& Pishkin, 1975). Moreover, it can be demonstrated regardless of whether or not the spatial location is the relevant cue.

According to one interpretation, the faster RTs observed in the more compatible $S-R$ pairings are attributed to a "basic natural tendency to respond toward the source of stimulation" (Craft \& Simon, 1970 , p. 419). The emphasis is on the comparable relation between concurrent stimuli and motor activities. This emphasis on motor output was challenged by Wallace's (1971) finding that compatibility effects were obtained between the position of the stimulus (left or right) and that of the response key, whether or not the subject's hands were crossed. Apparently, the effect does not depend on the relation between a stimulus and a particular motor output. Wallace (1971) hypothesized that the positions of both the stimulus and the responding hand were related to a spatial code, and that it was the outcome of a comparison between their representations in this code that was responsible for the differences in RTs.

It should be noted that an explanation of the S-R compatibility effect in terms of relations between stimuli, rather than between stimulus and response, entails a presumption that one of the stimuli must be the hand itself. As Wallace (1971) put it, "In other words, if subject's hand is in a position in space which is similar to that of the figure, the match between these positions is related to this hand and thence to the output involving the muscles of that hand and limb" (p. 159).
However, the difficulty associated with this explanation lies in the fact that the S-R compatibility effect can be demonstrated without the use of a manual response. For example, Bertera et al. (1975) measured eye movement RT in a choice RT situation and found that oculomotor RTs were faster when the tones signaling right or left looking were presented in the ear corresponding to the direction of eye movement (oculomotor response) than when they occurred in the opposite ear. Since there was no other stimulus than the tone itself in this situation, it is difficult to attribute the facilitating effect to the S-S compatibility, as claimed by Wallace (1971).

However, objections can be raised against the direct comparisons between the oculomotor and the manual responses. For example, since the former differs widely from the latter in both anatomical organization and operational characteristics, it could be argued that different mechanisms are responsible for the obtained compatibility effects in the oculomotor and the manual systems, even if the effects are seemingly similar. Thus, Wallace's (1971) S-S interpretation may still be correct within the manual system. To avoid such an argument, the two experiments reported here measured the manual RTs to visual signals presented in various spatial locations. Only a single hand was employed to make different responses, such that the hand as a stimulus was constant in all response conditions. Experiment 1 investigated whether the S-R compatibility effect would be present when choice responses were all produced by the same hand.

\section{EXPERIMENT 1}

\section{Method}

Subjects. Sixteen University of California at Riverside students with normal or corrected-to-normal vision participated in the experiment. All subjects were right-handed as assessed by 
self-report. All subjects were naive to the purposes of the experiment.

Apparatus. Four diffuse red light-emitting diodes (LEDs) subtending a visual angle of about $8 \mathrm{~min}$ were mounted in a Scientific Prototype tachistoscope. The LEDs were $1.5 \mathrm{deg}$ to the left or right and $1 \mathrm{deg}$ above or below a fixation point (see Figure 1). The fixation point consisted of a red point source LED subtending a visual angle of about $2 \mathrm{~min}$. Subjects responded to the stimulus display by depressing one of two telegraph keys $3 / 4$ in. to the left or right of a key upon which the subject centered his or her forefinger. The center key also served as a start key. Interval timing was achieved with Scientific Prototype and Lafayette interval generators. Response times were recorded on a Hunter Klockounter (Model 220C).

Procedure. Each trial began with the sounding of a tone by the experimenter, indicating that the subject might initiate a trial by depressing the start key which produced a fixation point lasting for $1 \mathrm{sec}$. A stimulus LED was then presented for $2 \mathrm{sec}, 100 \mathrm{msec}$ after the offset of the fixation point. The subject was instructed to indicate as quickly as possible whether the stimulus light occurred above or below the fixation point by depressing the response key to the left or right of the start key with the forefinger of a specified hand. After recording the response time, the experimenter sounded the tone to initiate the next trial. Trials were delivered in two blocks, each consisting of 16 warmup and 48 experimental trials. Thus, 12 trials were presented at each of the four stimulus locations for each block. Subjects used either the left or right hand in the first block and the opposite hand in the second block. Order of hand use was randomized across subjects.

Half of the subjects were instructed to press the right response key if a stimulus onset occurred above the fixation point and to press the left key if an onset occurred below the fixation point. The remaining subjects were given the opposite instructions, that is, to press the left key if a stimulus was presented above the fixation point, etc.

\section{Results and Discussion}

Mean correct response times were calculated for each of the stimulus and response variables. A 2 by 2 by 2 by 2 by 2 within-subjects ANOVA was performed on the following variables: responding with the left or right hand, position of the stimulus onset above or below the fixation point, stimulus presentation in the left or right visual field, order of hand use, and instructions to subjects to respond left or right for a stimulus onset occurring above the fixation point.

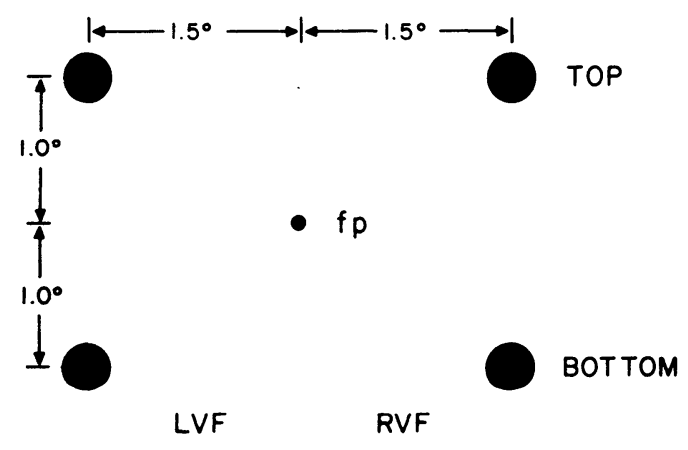

Figure 1. Layout of the stimulus pattern (four possible locations) and the fixation point (fp).

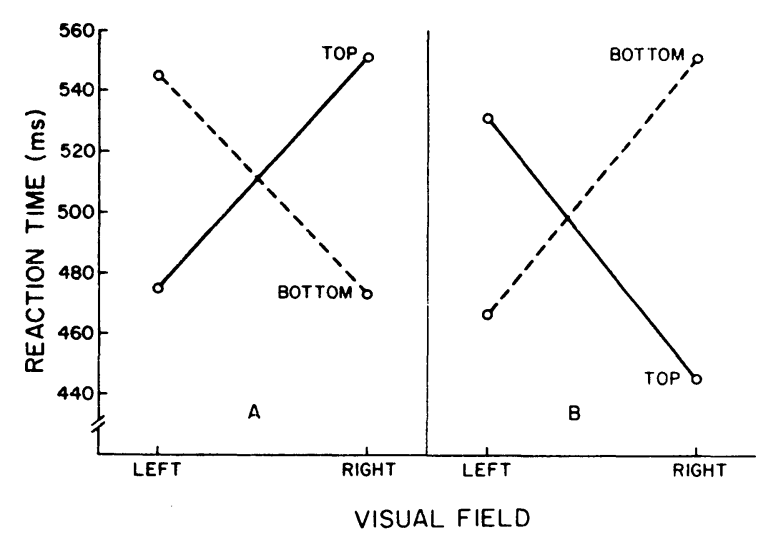

Figure 2. Stimulus-response compatibility effects as a function of response instruction. For the results shown in Panel A, subjects were instructed to press the left response key if a top stimulus occurred and the right key if a bottom stimulus was presented. For the results depicted in Panel B, the response instructions were reversed.

The results of Experiment 1 are shown in Figure 2. None of the main effects was found to be significant. The results indicate that when a single hand is employed in responding, S-R compatibility effects are still found. The interaction of Response Instruction by Stimulus Position by Visual Field indicated that, when the stimulus position corresponded to the position of the signaled response, response times were shorter than when it occurred in the opposite position $[\mathrm{F}(1,8)=$ 104.97, $\mathrm{p}<.001$ ].

The pattern of response latencies was similar to that reported by Bertera et al. (1975). Clearly, differential stimuli associated with each of the two response hands are not necessary for the observance of this phenomenon.

The results are consistent with an explanation of compatibility effects based on a tendency to respond to the source of stimulation proposed by Craft and Simon (1970). They proposed that when the locus of the signaling stimulus does not correspond to the locus of the required response, a response toward the source of the signal must be inhibited before the appropriate response can be initiated. According to this explanation, in Experiment 1 a stimulus presented to the left of fixation which commanded a response to the right produced an initial tendency to make a response corresponding to the locus of the stimulus (i.e., left) which had to be inhibited before the correct response could be produced.

In Experiment 2 the response keys were situated such that the required responses were located symmetrically with respect to the locus of the stimuli occurring to the left and right of fixation. Should the explanation proposed by Craft and Simon (1970) be correct, it would be expected that no compatibility effects would be found. 


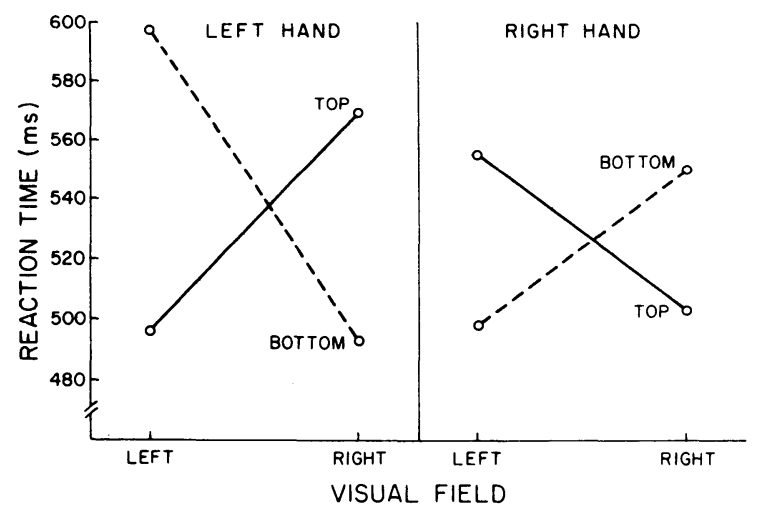

Figure 3. Reaction times as a function of stimulus position (top or bottom), visual field, and response hand.

\section{EXPERIMENT 2}

\section{Method}

Subjects. Sixteen subjects meeting the same criteria as before participated in the experiment.

Apparatus. For this experiment the response board was rotated 90 deg so that the response keys were above and below the start key.

Procedure. Subjects were instructed to press the top key if a stimulus onset occurred above the fixation point and the bottom key if the stimulus onset occurred below fixation. The rest of the procedure remained the same.

\section{Results and Discussion}

Mean correct response times were calculated for each of the stimulus and response conditions. A 2 by 2 by 2 by 2 repeated measures ANOVA was performed for the following variables: responding with the left or right hand, position of the stimulus above or below fixation, stimulus presentation in the left or right visual field, and order of hand use.

The results of Experiment 2 are shown in Figure 3. None of the main effects was found to be significant. An interaction of Response Hand by Stimulus Position by Visual Field indicated that response times were shortest when a top stimulus was presented to the visual field corresponding to the response hand and when a bottom stimulus was presented to the opposite visual field $[F(1,14)=114.85, p<.001]$.

Even though the correspondence between the stimulus and response locations was greatly increased, differential patterns of response latencies were associated with the four stimulus locations. If it may be assumed that in psychological space the left and right stimulus positions were situated symmetrically with respect to the response keys, no compatibility effects would be predicted by an explanation based on S-R location correspondence. Nor can these findings be interpreted in terms of a stimulus location and response hand correspondence explanation, since equally short response times were found for stimuli presented to both visual fields for a given response hand.

Although Experiment 2 is procedurally analogous to S-R compatibility experiments, the topography of responding was manipulated such that any compatibility effects should have been eliminated, yet a somewhat similar pattern of results was obtained. Since current conceptualizations of the nature of S-R compatibility effects cannot adequately explain this phenomenon, it would appear that our findings must be dealt with by some other paradigmatic model. A series of experiments is currently in progress to further investigate this phenomenon. Any explanation capable of dealing with our findings may also be relevant to those of the more general S-R compatibility paradigm.

\section{REFERENCES}

Bertera, J. H., Callan, J. R., Parsons, O. A., \& Pishein, V. Lateral stimulus-response compatibility effects in the oculomotor system. Acta Psychologica, 1975, 39, 175-181.

CRAfT. J. L.. \& Simon. R. Processing symbolic information from a visual display: Interference from an irrelevant directional cue. Jourmal of Experimental Psychology, 1970, 83, 415-420.

Raввітt. P. M. Signal discriminability, S-R compatibility and choice reaction time. Psychonomic Science, 1967, 7, 419-420.

Simon. J. R., \& Wolf, J. D. Choice reaction time as a function of angular stimulus-response correspondence and age. Ergonomics, 1963, 6. 99-105.

Wallace. R. J. S-R compatibility and the idea of a response code. Journal of Experimental Psychology, 1971, 88. 354-360. 